\title{
Common Medical Disorders Related To Diving - Prevention, Diagnosis And Fundamentals Of Treatment. Part 1: Diving Disorders That Do Not Require Recompression.
} Extracts from Institute of Naval Medicine Report R98013,
The Prevention and Management of Diving Accidents

Edited by DE Ayers, SJ Mercer, M Glover

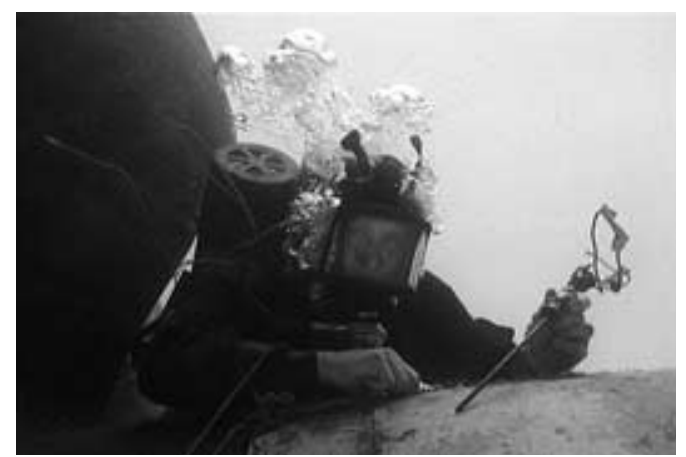

\section{Introduction}

This paper, which is published in two parts, is aimed at the non-specialist and reviews in outline the more common medical disorders related to diving. A more in-depth account can be found in the medical section of BR2806, the UK Military Diving Manual, and Institute of Naval Medicine Report R988013, The Prevention and Management of Diving Accidents (1). The latter work, from which this paper has been extracted, contains the distilled wisdom of generations of Royal Navy divers and diving medical officers including exchange officers from allied Navies. This paper is intended as an introduction for those who may infrequently encounter these conditions, rather than a scientific treatise and so is deliberately devoid of further references. It is no substitute, however, for more detailed and systematic study and the Royal Navy's Standard Underwater Medicine Course is commended to readers with an interest.

The work has been divided into two parts - diving disorders that do not and those that do require recompression, the latter to be published in a subsequent edition of The Journal. The work focuses principally on disorders resulting from the use of open circuit breathing apparatus in which the diver does not rebreathe any previously exhaled gas. Closed circuit systems do have a number of uses in the military setting but are generally beyond the scope of this work. Both sections aim to explain the mechanisms of the conditions covered, their recognition and initial management as well as the necessity or otherwise of referral. The details of therapeutic recompression have not been covered, with emphasis instead placed (in Part II of the paper) on recognising the need for recompression.

\section{Part I - Diving disorders that do not require recompression}

This section covers diving related illnesses and injuries not usually requiring therapeutic recompression. As general principles, any diver should either not dive or not remain in the water if they feel unwell, and any illness that occurs during or after a dive should be considered to be due to that dive until proven otherwise (Table 1).

\section{Oxygen deficiency (hypoxia/anoxia)}

This condition is rare when diving on air using open circuit breathing apparatus, but if it occurs it is either due to interruption of the breathing gas supply, or normal delivery but with insufficient oxygen in the gas mixture. In cases of inadequate supply such as when running low on air using SCUBA equipment, the diver notices increased work of breathing before being overtaken by the symptoms of hypoxia. If the breathing gas is of adequate volume but contains insufficient oxygen, the diver may notice few symptoms before losing consciousness other than a sense of well-being. Loss of judgement may, however, be apparent to the victim's buddy. The treatment is to restore interrupted supply or switch to another supply and administer $100 \%$ oxygen when on the surface.

\section{Oxygen toxicity}

If oxygen is breathed at a high partial pressure for long periods, it becomes toxic to many tissues, particularly the lungs and central nervous system.

\section{Pulmonary oxygen toxicity}

At partial pressures greater than 0.5 
Atmospheres Absolute (ATA) oxygen will become toxic to the lungs. For practical purposes this does not arise from normal air 'bounce' diving (i.e. where no planned recompression is required after the dive), to less than 50 metres of seawater (msw), because decompression considerations limit the period of exposure to oxygen within safe limits. It can occur where pure oxygen closed breathing sets are used and manifests as tickly throat, coughing then substernal burning. Advanced cases will manifest with physical signs and impaired lung function but, in most cases, complete recovery can usually be expected. The treatment is simply to reduce the concentration of oxygen in the mixture, preferably to 0.2 atmospheres.

\section{Cerebral oxygen toxicity}

Cerebral toxicity can be highly variable in its presentation with signs and symptoms including dizziness, nausea, tunnel vision and convulsion. Furthermore, there is no fixed oxygen exposure at which toxicity occurs with susceptibility varying both between individuals and within the same person from day to day. In most underwater breathing apparatus the inspired partial pressure of oxygen is limited to 1.4 ATA or less in order to make the risk tolerable. The first sign of cerebral oxygen toxicity can be generalised tonic-clonic seizure. If so, during the tonic phase, which lasts up to a minute, it is dangerous to surface the casualty as spasm of the glottis can result in inadequate exhalation, lung overpressure and consequent pulmonary barotrauma. If this occurs underwater the treatment is to keep the diver's depth as constant as possible in the tonic phase and then return to the surface. In the clonic phase the victim undergoes true convulsions. Once at the surface, breathing apparatus should be removed and the casualty placed in the recovery position in fresh air to recover. If further convulsions occur the airway should be protected. All cases should be kept under observation for twelve hours to ensure that the seizures are not due to some cause other than oxygen toxicity. If an uncontrolled ascent is made, an examination, including a neurological examination, must be carried out to exclude pulmonary barotrauma and decompression illness (see below). Loss of memory almost always occurs but is usually short-term and resolves quickly.

\section{Carbon dioxide poisoning (hypercapnia)}

Carbon dioxide poisoning may occur with or without a deficiency of oxygen. It may be caused whilst using open circuit apparatus by incorrect or shallow breathing, by excessive resistance or dead space within the equipment, or by using dense gases such as air or nitrox (nitrogen/oxygen mixture) at depths in excess of $30 \mathrm{msw}$. It manifests as progressive shortness of breath, nausea, headache, dizziness, neuromuscular twitching, convulsions and eventually loss of consciousness. If it occurs at depth the diver should reduce his activity and ensure that there are no problems with his gas supply. Once at the surface recovery should be rapid, but a throbbing headache is a frequent after-effect.

\section{Carbon dioxide deficiency (hypocapnia)}

This results from voluntary hyperventilation prior to a breath-hold dive in an attempt to extend endurance or from involuntary overbreathing while undertaking a dive. It may be provoked by anxiety states or fear. Common symptoms include dizziness, poor coordination, headache and classical carpopedal spasm with peri-oral paraesthesia. It should be noted that the symptoms of hypocapnia can be very similar to some of the constitutional and neurological manifestations of decompression illness and the distinction, though difficult, needs to be made correctly. Once recognised, most cases of hypocapnia can be successfully managed by voluntary regulation of the breathing rate. If symptoms persist despite attempts to control this, the time-honoured treatment of breathing from a paper bag will alleviate symptoms.

\section{Nitrogen narcosis ('narcs')}

If nitrogen is breathed under pressure, it acts like an anaesthetic and induces narcosis. The severity of effect depends on the depth of the dive, the rate of compression and the experience of the diver. Some drugs, particularly alcohol and sedatives, may have an additive effect to the narcosis and should not be taken prior to diving. Other gases also have a narcotic effect, the narcotic potency being proportional to their lipid solubility. Individuals vary in their susceptibility to nitrogen narcosis and some behavioural adaptation to the effects of narcosis can be achieved by frequent exposure. The effect varies with depth, ranging from lightheadedness and euphoria at 30-60 msw through progressively poorer judgement and hallucinations to eventual loss of consciousness at more than $120 \mathrm{msw}$. The symptoms and signs of nitrogen narcosis are similar to those of drunkenness except that there is no hangover. There is no danger from the narcotic effect itself and the effect wears off rapidly on reducing depth. The hazard is that a narcosed diver may act inappropriately, sustain an injury or drown while his concentration is impaired.

Nitrogen narcosis is prevented by limiting the depth of dives according to the experience of the diver. Deep dives are 
preceded by a work-up, which does not prevent narcosis, but enables the diver to learn to cope with the effect. Deeper dives are conducted using helium rather than nitrogen as the inert gas in the breathing mixture. If narcosis occurs, treatment is to reduce the depth of the dive and in rare serious cases the diver should surface. Once the inspired pressure of nitrogen is reduced, the effects of narcosis resolve rapidly.

\section{Caustic cocktail}

Despite this article's focus on open circuit diving systems, one important and dramatic condition particular to closedcircuit systems needs to be considered. If water enters a closed circuit breathing apparatus it might mix with the carbon dioxide absorbent (usually a mixture of calcium hydroxide with a small amount of sodium hydroxide). This strong alkali solution can cause severe chemical burns to the face, mouth, lungs and stomach. In order to prevent this, equipment needs to be checked for leaks before diving and if one becomes apparent during a dive then the diver needs to surface as soon as he is able. The diver should leave the water and remove the set as quickly as possible. The oropharynx should be inspected for signs of burning and, if apparent, the victim should wash his mouth out with fresh water and drink more to dilute the alkali. If salt water is all that is available at the time, then this may be used to wash out the mouth, but should not be swallowed as this may induce vomiting. Vomiting is dangerous and can cause further burning to the oesophagus and oropharynx if the caustic cocktail has been swallowed. Any skin surface exposed to the injurious agent should be washed liberally with fresh or salt water.

Any patient with known or suspected oropharyngeal burns should be referred urgently for endoscopy. Caustic burns to the eye should similarly be treated by washing with large volumes of water and subsequent referral to an ophthalmologist. Caustic cocktail readily provokes panic which may result in rapid ascent and breath holding (to avoid inhaling further alkali). Consequently, divers who have accidentally inhaled a caustic cocktail should be carefully observed following the dive for evidence of pulmonary barotrauma or neurological decompression illness.

Table 1. Conditions caused by effects of $\mathrm{O}_{2}, \mathrm{CO}_{2}$ and $\mathrm{N}_{2}$.

\begin{tabular}{|l|l|}
\hline Oxygen deficiency & $\begin{array}{l}\text { Increased work of breathing, inappropriate sense } \\
\text { of well-being }\end{array}$ \\
\hline Oxygen toxicity & $\begin{array}{l}\text { Dizziness, nausea, tunnel vision, generalized } \\
\text { convulsions }\end{array}$ \\
\hline $\begin{array}{l}\text { Carbon dioxide } \\
\text { toxicity }\end{array}$ & $\begin{array}{l}\text { Progressive shortness of breath, nausea, dizziness, } \\
\text { headache, eventual loss of consciousness }\end{array}$ \\
\hline Nitrogen narcosis & $\begin{array}{l}\text { Light-headedness and euphoria, poor judgement, } \\
\text { hallucinations, eventual loss of consciousness }\end{array}$ \\
\hline
\end{tabular}

\section{Compression barotrauma (squeeze)}

The body is mainly composed of incompressible fluid. However, any gasfilled space present within or next to the body can damage tissues when its volume changes in accordance with Boyle's Law. 'Squeeze' refers to the damaging effect of a reduction in volume of gas in a diver's equipment or body cavity as pressure is increased. The opposite effect, barotrauma of ascent, is caused by the expansion of gas. The predominant symptom of barotrauma is pain. In situations where the gas volume can be restored to the pre-dive volume, e.g. by inflating a dry suit or 'clearing' the ears, squeeze can be avoided. Equally, where the gas is contained within a flexible viscus such as the bowel, compression and subsequent expansion of gas can occur harmlessly. The types of squeeze most frequently encountered in diving are in the ear, suit, sinuses, lung, facemask and tooth.

Suit squeeze is caused when a pocket of air under a fold or fitting of a dry suit is compressed and results in the skin being pinched. Sinus squeeze may occur when the passages that vent the sinuses into the nasal cavity are obstructed. It presents as pain because the pressure in the affected sinus decreases relative to ambient, causing oedema of the mucosa and possibly haemorrhage into the space. Lung (thoracic) squeeze is a very rare event, which may occur if the air in the lungs is compressed to a volume somewhat less than the residual volume of the lungs. Facemask squeeze is caused by a failure to equalise air pressure in the mask by nasal exhalation. Facial oedema over the area covered by the mask as well as subconjunctival haemorrhages may result. Tooth squeeze results when a small pocket of trapped gas exists under a poorly prepared or cracked filling. If it is completely isolated, the pulp of the tooth or the tissues of the socket can be drawn into the space causing pain. The treatment of all of these instances of barotrauma is to stop the descent and if efforts to equalise the pressure fail, ascend to a shallower depth.

\section{External ear squeeze - 'reversed ears'}

External ear squeeze or 'reversed ears' occurs when the external auditory meatus (EAM) is blocked by an obstruction such as wax, a tight fitting hood, earplugs or otitis externa and the pressure in the outer ear cannot equilibrate with the ambient pressure. During descent therefore, a relative vacuum develops in both the outer and middle ear. When the ears are cleared, which returns the middle ear pressure to ambient, the tympanic membrane balloons outwards, in the opposite direction to that 
which occurs in more conventional middle ear barotrauma - hence 'reversed ears'. If it persists for more than a few minutes, then injury to the epithelial lining of the EAM and tympanum may occur with oedema and petechial haemorrhages. Accordingly, divers should be told not to dive if they have an outer ear infection and the use of tight fitting hoods and earplugs should be avoided. The condition is heralded by the onset of pain similar to that which occurs in the more classic middle ear barotrauma described below. There may also be mild vertigo and on examination the external canal may be swollen or haemorrhagic. Patients with reversed ears should be referred to an otolaryngologist especially if tympanic rupture is suspected. Eardrops, which may be ototoxic, should be avoided.

\section{Middle ear barotrauma ('ears')}

Middle ear barotrauma is due to a failure to equalise middle ear pressure with ambient and remains the most common medical problem associated with diving. It is both painful and potentially dangerous.

The eardrum completely seals off the outer ear canal from the middle ear space and when the diver descends, the pressure on the outer surface of the drum increases. To maintain the status quo, the air pressure on the inner surface of the drum must increase by the same amount. This is accomplished by the diver allowing air to pass down the Eustachian tube, which connects the pharynx to the middle ear (see Figure 1). This process is known as 'clearing the ears'. In a few divers, the Eustachian tube is open all the time, so no conscious effort is necessary to clear their ears. For most, however, the Eustachian tube is naturally closed and some action must be taken to allow this passage of air. Many can accomplish this by yawning, swallowing or

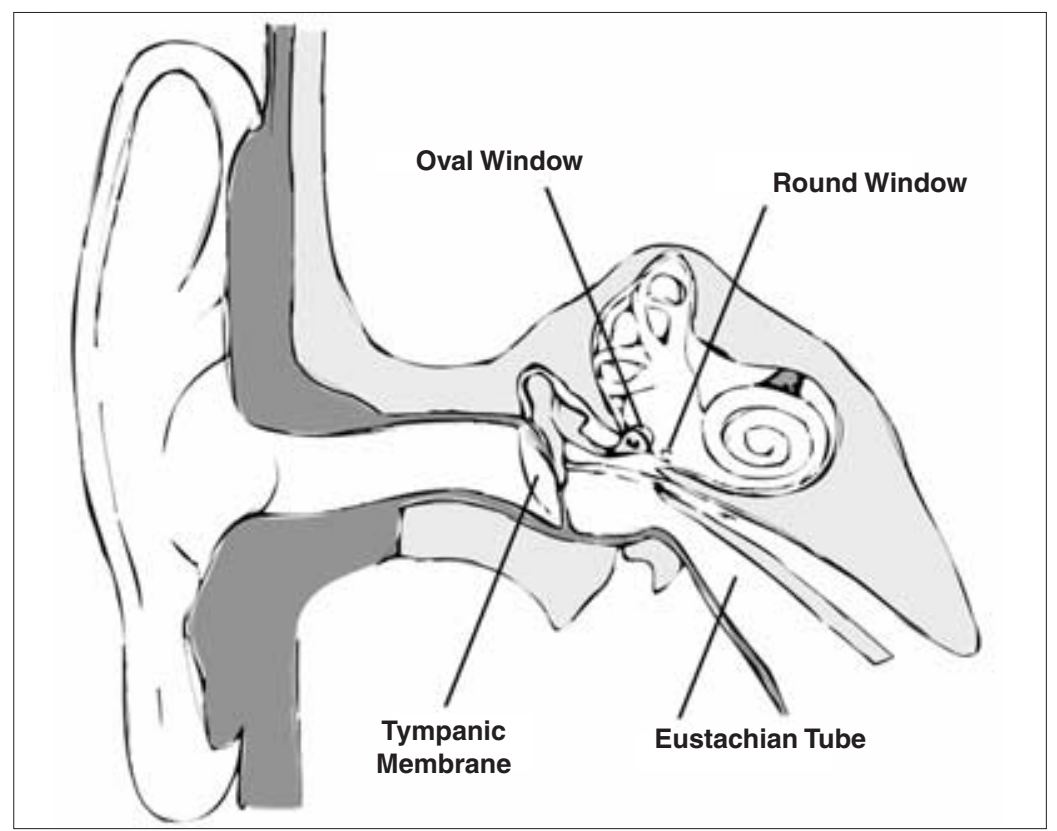

Fig1. A coronal section through the middle and inner ear. moving the jaw around. Some require a Valsalva manoeuvre against a closed nose and mouth (rather than a closed glottis) to achieve this.

If the Eustachian tube is blocked, the ears cannot be cleared. During the initial phase of descent, the decreasing volume of the middle ear is compensated for by the drum being displaced inwards. Eventually, however, the eardrum reaches the limit of its inward distensibility and the middle ear pressure falls below the external water pressure. This creates a relative vacuum in the middle ear space and so a pressure difference across the tympanic, round and oval window membranes. This negative pressure causes the blood vessels of the eardrum and the lining of the middle ear to expand, leak and eventually rupture. If descent is continued, then either the eardrum will rupture allowing the immediate equalisation of the middle ear and ambient pressure, or bleeding into the middle ear becomes sufficient to equalise the pressure by replacing air with incompressible fluid.

The first symptoms are pressure and fullness in the ear followed by pain, usually experienced within the first 6-7 $\mathrm{msw}$ of descent (Table 3). If the tympanic membrane ruptures, relief of pain occurs almost immediately. If this results in ingress of water to the middle ear, caloric vertigo due to thermal imbalance between the two ears may occur. Following the dive there may be some residual pain and a mild conductive hearing loss and the ears should be examined with an otoscope. In milder cases, signs range from a normal otoscopic appearance through to redness and retraction of the membrane, possibly with haemorrhage within the membrane. In more severe cases, a bulging membrane with free blood in the middle ear or tympanic membrane perforation with free haemorrhage is seen. Hearing loss usually recovers within two days but, if persistent, ossicular chain disruption may have occurred.

In order to prevent middle ear barotrauma, divers should avoid diving with a cold or nasal congestion, since the Eustachian tube may be blocked. Divers who cannot clear their ears on the surface should not dive. A diver who has suffered middle ear squeeze should not return to diving until healing is complete. If there is bleeding from the ear or vertigo following a dive, the medical officer should refer the diver to an otolaryngologist. Again, eardrops which contain drugs that may be ototoxic should not be used if a perforated tympanic membrane is seen or suspected.

\section{Alternobaric vertigo}

This condition occurs quite commonly. It arises when either during descent, or more 
Table 2. Conditions caused by pressure changes.

\begin{tabular}{|l|l|}
\hline $\begin{array}{l}\text { Middle ear barotrauma } \\
\text { and reversed ears }\end{array}$ & $\begin{array}{l}\text { Pressure and fullness in ears, } \\
\text { increasing pain in ears }\end{array}$ \\
\hline Tooth squeeze & Pain in tooth on changing depth \\
\hline Sinus squeeze & Headache or face pain on changing depth \\
\hline Alternobaric vertigo & Transient dizziness and nausea \\
\hline
\end{tabular}

commonly ascent and (particularly when in the upright position), the change of middle ear pressure between the two sides is asymmetrical. This can result in a transient sensation of vertigo, disorientation or nausea. If it occurs at depth, it can be hazardous. These symptoms normally resolve rapidly once the diver equalises pressure in the affected middle ear.

\section{Caloric Vertigo}

Usually on immersion, water enters both ears and the inner ear is cooled at a similar rate on each side. Caloric vertigo, which has been mentioned already, occurs when one ear is partially or completely blocked by a tight fitting hood or wax and the inner ears are stimulated unequally. Vertigo, which tends to be transient, results.

\section{Otitis externa (swimmer's ear)}

Repeated immersion breaks down the skin which lines the external ear canal and this allows the bacteria and fungi normally present to multiply. The diver complains of itching, a wet feeling or discharge and, if severe, pain. It is a completely preventable condition. 7\% Aluminium acetate solution or Otic Domeboro ${ }^{\circledR}$ ear drops prevent infection if applied after each wet dive. These solutions are both bacteriostatic and astringent and should be left in for a minimum of 5 minutes. If the condition is contracted, treatment is a temporary cessation of diving so that the ear can be kept dry and continued use of aluminium acetate drops and appropriate antibiotic drops if necessary.

\section{Near drowning}

Drowning is the most common cause of diving fatalities. Divers drown as a result of accidents, panic, over-exertion or the effects of cold. Near drowning describes the condition of those who survive such submersion - at least, temporarily. The victim is unconscious, often the body is leaden-blue in colour and froth is visible around the nose and mouth. It should be noted that in $10-15 \%$ of cases water does not enter the lungs due to the effects of laryngospasm. The mouth and throat should be cleared of debris and cardio-pulmonary resuscitation (CPR) commenced. Time should not be wasted trying to drain the lungs but oxygen should be administered if available. The victims of near drowning are often severely hypothermic and may appear dead. Such victims should not be considered dead until they are completely re-warmed and continue to be unresponsive to resuscitation.

A person with water in his lungs may not lose consciousness immediately, but may still die from later 'secondary drowning' due to pulmonary oedema. This can result from reaction to the inhalation of either fresh or salt water. For this reason all suspected near drowning victims should be observed in hospital for 24 hours.

\section{Vomiting underwater}

This is an extremely dangerous and potentially life threatening event. It may be caused by diving with a gastro-intestinal upset, over exertion after recent intake of alcohol or food, or as part of another underwater incident such as ingestion of caustic cocktail. The correct management is to get the diver out of the water as soon as possible and remove the breathing apparatus. If unconscious, the airway should be cleared and CPR begun if necessary.

\section{Underwater blast injury}

Proximity to underwater explosions is dangerous and the resultant injuries are complex. In short, shrapnel injuries are unusual as material displaced by the blast does not travel far. The pressure waves propagate much further and release energy at density interfaces. Energy of a pressure wave is transmitted to the tissues of the body more efficiently from water than from air because of the similarity in densities. There is significant disruption, however, of tissues containing gas as destructive energy is released due to the sharp density changes at gas-tissue interfaces and the spaces distort with the pressure changes. Accordingly, perforated eardrums may be seen as well as pain or haemoptysis from injuries to bowel or lungs. Casualties should be assessed and treated for immediate life threatening conditions in accordance with basic principles and evacuated immediately for more detailed assessment.

\section{Cold exposure and hypothermia}

Cold immersion is thought of as acute and sub-acute. Acute cold immersion results from sudden unprotected entry into cold water or the rapid influx of cold water into a diving suit. As well as immediate increases in pulse and blood pressure this results in a period of rapid uncontrolled breathing. As divers usually enter the water protected and in a controlled fashion, this acute cold reaction is rare. Immersion in water below about $32^{\circ} \mathrm{C}$ however, soon results in the unprotected diver becoming chilled. Discomfort is followed by shivering and loss of first manual then mental dexterity. Thus the diver may make mistakes which themselves could be fatal. If immersion is 
prolonged, heat loss reaches a point where death occurs from hypothermia. The symptoms and signs of a falling core temperature begin with pale skin and sporadic shivering at around $36^{\circ} \mathrm{C}$, which then becomes uncontrollable (Figure 2). Mental confusion takes hold around $35^{\circ} \mathrm{C}$ and the diver is at risk of drowning. Gradually speech is impaired and consciousness clouds when the core reaches $33^{\circ} \mathrm{C}$. Evidence from shipwrecks suggests that $50 \%$ of individuals will not survive this stage. Shivering may cease as the casualty cools further and the heart rhythm may become irregular. Loss of consciousness occurs at around $30^{\circ} \mathrm{C}$ and nearly all are dead by $27^{\circ} \mathrm{C}$. Treatment of the casualty begins with prevention of further heat loss by removing the casualty from the water, maintaining them throughout in a horizontal position to prevent cardiovascular collapse. The patient then needs to be gently re-warmed, ideally in a medical facility, and CPR instituted if necessary. Caution should be exercised in making the decision to commence cardiac massage, as it can induce arrhythmias and will need to be continued until re-warming is complete. Milder cases of hypothermia where consciousness has been maintained throughout can be re-warmed by careful immersion in a bath at $40^{\circ} \mathrm{C}$.

\section{Heat exhaustion}

This occurs as a result of over-exertion by a diver in warm water (over $25^{\circ} \mathrm{C}$ ), when wearing either a dry suit or a wet suit that is too thick, or normal levels of exertion in water over $30^{\circ} \mathrm{C}$. This should be a preventable condition but, when it does occur, manifests as dizziness and headache with a rapid pulse and a raised core temperature. Usually, but not always, there is excessive sweating. As the temperature rises further, conscious level is reduced and coma supervenes. The patient should be rested in a cool place, encouraged to drink water and cooled by evaporative heat loss through wetting and fanning. All but the mildest cases should be transferred to hospital as the condition can readily and sometimes rapidly deteriorate to lifethreatening severity. It can take over 24 hours for spontaneous thermoregulation to be restored.

\section{Decompression pulmonary barotrauma}

Decompression pulmonary barotrauma is a syndrome that results in gas, which is normally retained within the airways, leaving its natural confines and entering either the interstitial space within the lung, the pleural cavity or the blood stream. The mechanisms by which this happens are incompletely understood. At the simplest level, if gas, which has been breathed while at depth, is trapped within the lung during ascent, then the resulting expansion in volume of that gas, in accordance with Boyle's Law, may be sufficient to cause delicate pulmonary tissue to rupture. The gas may be trapped as a result of voluntary or involuntary breath holding, or as a result of pulmonary pathology. However, numerous cases of decompression pulmonary barotrauma have occurred in which no evidence of pulmonary pathology has been found and exhalation during ascent has been witnessed by independent observers. Consequently there probably remain other causes of the condition that have yet to be identified.

Divers should be screened, therefore, for evidence of obstructive lung disease which could result in trapping of gas. Those suffering short-term respiratory illness should not dive while they remain

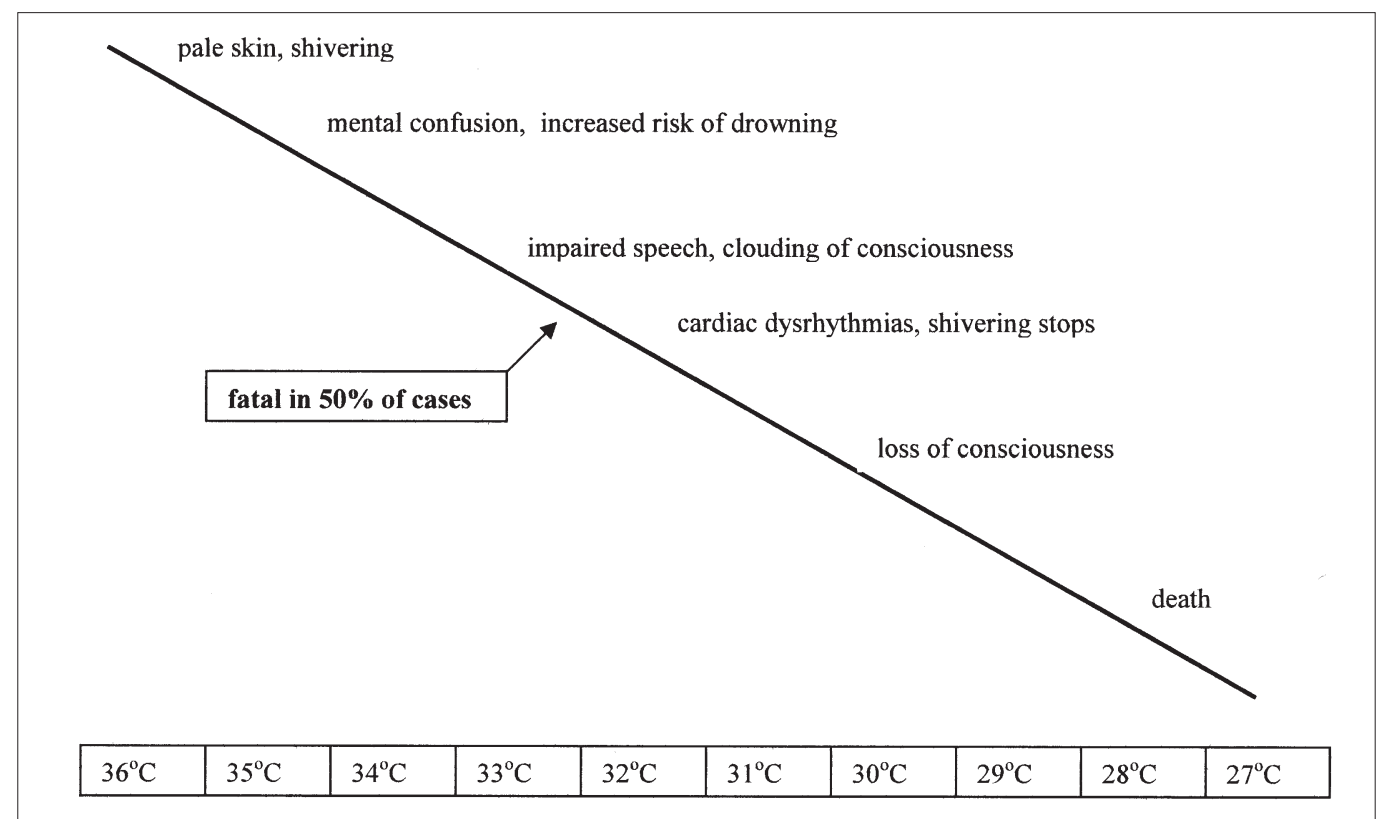

Fig 2. Symptoms of reducing core temperature. 
symptomatic. Decompression pulmonary barotrauma manifests with a range of symptoms and signs. Certain of these are usual, such as sharp retrosternal chest pain, shortness of breath, painful breathing and productive cough with small amounts of blood staining in the sputum (Table 3). Others depend on the route the gas takes after rupture and so are less consistently observed.

\section{Mediastinal and subcutaneous emphysema}

If gas escapes into the interstitial tissue space, it can track out of the airways and vessels to the hila and thence to the mediastinum. This is often asymptomatic but if there is a substantial quantity, retrosternal pain may be felt. Gas in the mediastinum may migrate to the subcutaneous tissues of the neck where it manifests as crepitation. Rarely it may track down to the abdomen where it lies in the retroperitoneum and though usually asymptomatic, may outline the liver or kidneys on plain radiography.

\section{Pneumothorax}

This occurs when alveolar gas escapes into the pleural space. It is often painless, but may cause a sharp pain, worse on deep inspiration. Commonly the pneumothorax is small with few physical signs, but a more substantial one demonstrates the classical signs of shortness of breath, cyanosis, reduced chest movement on and tracheal shift towards the affected side, hyperresonant percussion note and reduced breath sounds. If gas escapes into the pleural space with each breath but is unable to return a tension pneumothorax results. The danger is the resultant collapse of one, then both lungs. Tracheal shift is now away from the affected side, cyanosis is profound and shock, unconsciousness and death ensue if untreated. A simple pneumothorax which occurs at depth may increase in size during decompression and effectively become a tension pneumothorax. The diagnosis should, therefore, be suspected in a diver whose respiratory symptoms worsen during ascent.

Asymptomatic mediastinal and subcutaneous emphysema will resolve gradually without the need for any specific treatment. If there are troublesome symptoms then breathing $100 \%$ oxygen on the surface will accelerate resolution of symptoms. Small pneumothoraces can be treated just by

Table 3. Symptoms due to decompression barotraumas.

\begin{tabular}{|l|l|l|}
\hline Pulmonary & $\begin{array}{l}\text { Mediastinal or subcutaneous } \\
\text { emphysema }\end{array}$ & Retrosternal pain, skin crepitus \\
\hline & $\begin{array}{l}\text { Pneumothorax } \\
\text { Arterial gas embolus }\end{array}$ & $\begin{array}{l}\text { Shortness of breath, cyanosis, collapse. } \\
\text { Often painless } \\
\text { Cardiovascular collapse, neurological } \\
\text { disturbances }\end{array}$ \\
\hline Gastro-intestinal & Colicky abdominal pain, abdominal distension \\
\hline
\end{tabular}

administration of $100 \%$ oxygen, but large pneumothoraces will require draining. All tension pneumothoraces obviously require emergency decompression then siting of a chest drain.

\section{Arterial gas embolism (AGE)}

If gas from a ruptured lung enters the pulmonary veins, it will be carried to the left side of the heart and from there be distributed to the body as arterial gas emboli. The two organs that are particularly susceptible to functional disturbance as a result of such emboli are the brain and the heart. Divers sustaining decompression pulmonary barotrauma should, therefore, be examined for evidence of cardiac or cerebral dysfunction. In the past it was AGE in patients who experienced the onset of neurological symptoms during decompression or shortly after surfacing from a dive, even in the absence of any other evidence of pulmonary barotrauma. It is now recognised that such a diagnosis is liable to be inaccurate as other conditions can present in a similar manner. Accordingly, neurological symptoms or signs presenting after a hyperbaric exposure are termed 'neurological decompression illness' and are covered in the second part of this paper.

\section{Gastrointestinal barotrauma}

Gas normally present within the intestine expands during decompression and may result in eructation, flatus or abdominal discomfort. Occasionally such colicky abdominal pain and distension with 'tinkling' bowel sounds on auscultation, may mimic bowel obstruction, but will resolve spontaneously. If gas is swallowed (aerophagia) when under pressure however, it may be dangerous as more serious gastrointestinal barotrauma may result leading to rupture of a viscus during ascent.

\section{Conclusion}

This review, based on the medical section of the Royal Navy Diving Manual, represents current philosophy on the subject within the Royal Navy. It is intended as both a training and a practical aid, and has briefly covered diving related illnesses which do not usually require therapeutic recompression; part II of this review, to appear in a future issue of this journal, will cover diving disorders that require therapeutic recompression. considered possible to make a diagnosis of 
As a general rule, no diver should dive or remain in the water if they feel unwell, and any illness occurring during or after a dive should be considered to be related to that dive until proved otherwise.

\section{Acknowledgements}

We are indebted to those individuals who have contributed to the medical section of the Military Diving Manual and successive copies of Institute of Naval Medicine Reports on the Prevention and Management of Diving Accidents. Undersea Medicine Division of the Institute of Naval Medicine has provided advice and oversight to ensure that, despite considerable editing, this paper retains a faithful description of the principles of diving medicine and the problems covered by the original text from which it was drawn.

\section{References}

1. The Prevention and Management of Diving Accidents. Institute of Naval Medicine Report. No. R988013. 